Cahiers d'études africaines

168|2002

Musiques du monde

\title{
Réflexions sur un hymne continental
}

La musique africaine dans le monde*

Bob W. White

\section{(2) OpenEdition}

Journals

Édition électronique

URL : https://journals.openedition.org/etudesafricaines/159

DOI : 10.4000/etudesafricaines.159

ISSN : $1777-5353$

Éditeur

Éditions de l'EHESS

Édition imprimée

Date de publication : 1 janvier 2002

Pagination : 633-644

ISBN : 978-2-7132-1778-4

ISSN : 0008-0055

Référence électronique

Bob W. White, «Réflexions sur un hymne continental », Cahiers d'études africaines [En ligne], 168 | 2002, mis en ligne le 27 juin 2005, consulté le 01 juillet 2022. URL : http://journals.openedition.org/

etudesafricaines/159; DOI : https://doi.org/10.4000/etudesafricaines.159 


\section{Bob W. White}

\section{Réflexions sur un hymne continental}

La musique africaine dans le monde*

En dépit des appréhensions quant à son caractère imprévisible, la mondialisation commence à sembler drôlement prévisible. D'un côté, on applaudit la concrétisation du rêve néo-libéral de la déréglementation des marchés, de la libre circulation des capitaux et de l'accès illimité à une main-d'œuvre bon marché. De l'autre, on dénonce l'élargissement du fossé qui sépare les riches et les pauvres, les changements climatiques imminents et les menaces de disparition qui pèsent sur diverses espèces biologiques et culturelles. Cette inquiétude, comme l'observe Arjun Appadurai (2000) dans un ouvrage récent qui s'intitule tout simplement «Globalization », a servi les ambitions des industries du savoir en Occident, notamment la recherche et l'édition universitaires, sans pour autant les délivrer du mal du provincialisme. La couverture du livre de Appadurai, argentée et élégante, semble célébrer notre intérêt pour la mondialisation, mais, comme le montre l'ouvrage, il n'y a pas lieu de se réjouir. Nous continuons de parler de la mondialisation en des termes vagues et dualistes, comme une série de mauvaises choses arrivant à des gens bien. Comment peut-on raconter l'histoire de la mondialisation sans invoquer les formules fatiguées de l'angoisse ni se livrer à la négligence de la célébration ? (Feld 2000). Quelle est la couleur de cette mondialisation? Quel son produit-elle?

Une manière de répondre à ces questions est de les observer par le prisme du phénomène mondial de la world music. La plupart des observateurs s'accordent pour situer ses origines sur la scène de la musique alternative britannique et américaine des années 1980 (ibid.), mais ces discours sur les origines du phénomène donnent l'impression, souvent fausse, que

* Je tiens à remercier Marie-Nathalie Leblanc qui m'a fait connaître le travail de Yacouba Konaté et de Dorothea Schulz, et Bogumil Jewsiewicki pour son soutien dans les premières étapes de la préparation de ce numéro. Je suis aussi reconnaissant à mes anciens étudiants et collègues de l'Université de Californie (Santa Cruz) pour avoir stimulé ma réflexion sur ce sujet et sur d'autres sujets qui s'y rattachent. 
toute histoire a un début et une fin (Feld 1996; White ce numéro). Il est évident que la world music cherche à se manifester depuis bien longtemps, au moins depuis l'invention des nouvelles technologies de reproduction au début du dernier siècle, peu après la prise de conscience que les disques, loin d'être un simple moyen de vendre des phonographes, représentaient eux-mêmes une ressource lucrative et inépuisable. On ne sait pas grand chose des stratégies de commercialisation des premières sociétés de disques (Pekka \& Gronow 1998), mais il est évident qu'elles ont, très tôt, établi une distinction entre les disques destinés aux publics riches d'Europe et d'Amérique du Nord (Keightly 1998) et ceux — peut-être pas moins exotiques - destinés aux personnes souhaitant écouter le son de leur propre culture en dehors de l'Occident. Pour ne pas donner l'impression que l'histoire de la world music débute plutôt ici...

Avançons de 100 ans, à l'époque du glorieux retour de l'une des figures les plus révérées de la folk music américaine. Ce come-back coïncidait avec l'apogée de l'anomie musicale des années 1980 et la période vaguement militante du mouvement anti-apartheid. L'idiome musical par lequel il choisit de s'exprimer - la musique des travailleurs migrants en Afrique du sud - était certainement marqué par des nouveaux sons et des nouveaux rythmes, mais c'est avant tout le primitivisme reformulé de Paul Simon qui explique le succès connu par Graceland. En 1986, l'idée de world music était déjà dans l'air, mais c'est l'arrivée de cet album qui lui donna un nom. Plus qu'un simple album, Graceland a marqué toute une génération de musiciens et d'amateurs de musique nouvellement conscients de leur place dans le monde. "These are the days of miracle and wonder... this is the long-distance call... the way the camera follows us in slow-mo... these are the days of lasers in the jungle. " Pour certains critiques, cet album était un véritable tour de force ; pour d'autres, il incarnait la pire forme de néocolonialisme qui fût, et constituait une violation de l'embargo international imposé à l'époque contre l'Afrique du sud (Meintjes 1990 ; Hamm 1995). Bien sûr, les voyages d'autres icônes de la pop ont prouvé que Graceland était loin d'être un incident musical isolé : la collaboration de David Byrne et Brian Eno dans les années 1980 (Remain in Light et My Life in the Bush of Ghosts) s'est inspirée de plusieurs voyages en Afrique occidentale ; le manager des Sex Pistols, Malcolm McClaren, a foulé le même territoire musical que Paul Simon dans son album de 1984 intitulé Duck Rock, et l'ancien batteur du groupe The Police, Stewart Copeland, s'est associé au Zaïrois Ray Lema pour produire une fantaisie-safari de percussionniste dans son album The Rhythmatist.

Étant donné le rôle important joué par les vedettes occidentales dans l'articulation du genre, comment peut-on comprendre ces moments de traduction culturelle sans se fixer sur les traducteurs ? Une approche possible est de considérer la world music comme une catégorie de ce que Veit Erlmann (1996, 1999) appelle «l'imaginaire mondial». C'est l'approche choisie par un petit nombre d'ethnomusicologues qui se sont avant tout 
intéressés à la diffusion d'une musique tradi-moderne en provenance des régions du monde que nous sommes censés nous représenter comme pauvres sur le plan matériel, mais dotées d'une certaine richesse sur le plan spirituel (Falceto ce numéro ; Martin 2002). L'anthropologue Steven Feld a jeté les bases d'une approche critique qui établit un lien entre ce «nouveau » phénomène et la longue durée du primitivisme dans la pensée occidentale :

« Ces airs de world music, tout comme leurs prédécesseurs primitivistes ou nationalistes-romantiques, donc, se rapportent avant tout à l'exploration, au pouvoir et au privilège de contacter et de connaître, de prendre et d'utiliser... Comme d'autres variétés de primitivisme bien documentées dans d'autres domaines... la world music crée un voyage de découvertes, une expérience sonique de contact, une défloration auditive qui pénètre l'harmonie de la différence » (Feld 2000).

Pour reprendre la formule de Frederic Jameson, nous sommes confrontés, à n'en point douter, à une logique de capitalisme avancé qui se reproduit par la production de la différence (Erlmann 1993). «Le marketing est en soi une pratique fondée sur les différences, et plus ces différences sont nombreuses, plus les stratégies de marketing peuvent se développer » (Hardt \& Negri $2000: 152$ ). Mais un autre débat occupera une place beaucoup plus grande sur le plan intellectuel : c'est celui qui concerne la question de «agency »: «Les masses, écrit Veit Erlmann, finissent toujours par gagner, la consommation se transforme toujours en action, et le banal s'affirme comme un modèle utopique. » Jocelyne Guilbault (1996) adopte quant à elle une approche plus nuancée en explorant les moyens par lesquels ces catégories sont créées et activées, surtout dans le contexte professionnel de la discipline de l'ethnolomusicologie.

Il faut souligner qu'il existe (au moins) deux types d'ethnomusicologues : ceux qui utilisent le terme «world music » pour faire référence à la musique autre que classique provenant des pays non occidentaux, et ceux qui utilisent ce terme comme un aspect de l'imaginaire du consommateur occidental. Les premiers ont tendance à effectuer des recherches et à publier des articles destinés à la communauté de chercheurs en ethnomusicologie et certains aficionados (Charry 2000 ; Mallet ce numéro ; Zemp 1996). Les seconds ont un public beaucoup plus large, bien qu'essentiellement composé d'universitaires, et ont produit une quantité croissante d'études qui démontrent comment la vie quotidienne s'imbrique dans des économies politiques mondialisantes. La généalogie spirale de sons pygmées dans la musique pop occidentale réalisée par Feld (1996), l'étude révélatrice de Louise Meintjes sur les relations sociales et la réception de Graceland dans l'Afrique du Sud, les travaux encore non publiés de Chris Waterman sur l'histoire du mbube, et l'essai de Timothy Taylor (1997) sur l'authenticité ne sont que quelques exemples parmi les meilleurs. Le champ d'études consacré à la world music est en train d'émerger comme une discipline ethnographique non dépourvue de sens critique qui reste très proche à la question de la représentation (Born \& Hesmondhalgh 2000). 
Les articles figurant dans ce numéro reflètent une autre approche à l'étude de la world music. Cela est essentiellement dû au fait que la plupart des auteurs ont pris pour sujet d'étude les univers sociaux de l'expérience musicale quotidienne en Afrique plutôt que la réception de la musique africaine en Occident. Ils sont néanmoins décidés, comme l'était Jacques Attali (1977), à «non seulement théoriser sur la musique, mais à théoriser par la musique ». Ce qu'il y a de plus passionnant à publier une série d'articles sur la world music dans une revue d'études régionales est la possibilité d'examiner la manière dont les formes mondialisantes se reproduisent (ou ne se reproduisent pas) dans le contexte des économies locales et régionales. L'hégémonie des industries culturelles opère entre les différentes régions du monde mais aussi au sein de chacune d'entre elles (Manuel 1993 ; Guilbault 1993a; Pacini-Hernandez 1993), et les recherches à venir pourront améliorer notre compréhension de la production culturelle à plusieurs niveaux.

À l'exception de Julien Mallet, aucun des auteurs regroupés dans ce numéro spécial n'a entrepris d'écrire sur la «world music» dans le sens «Buenavistifié » dont je parle ci-dessus (Hernandez-Reguant 2000); aussi en apprenons-nous plus sur le monde de Fela Kuti (Veal 2000) que sur celui de Obo Addy (Taylor 1997). La tentative courageuse de Julien Mallet consistant à répertorier les catégories qui peuvent être qualifiées de world music ne fait que nous confirmer que, en tant que genre, la world music opère avant tout selon une règle d'exclusion (Martin 2002). Pourtant il y a dans la world music quelque chose d'identifiable qui transcende la contingence des étiquettes dans les magasins de disques. La motivation qui se cache derrière ces textes s'apparente plutôt à la notion de «branchements » développée par Jean-Loup Amselle (2001) qui procède de la volonté de comprendre qui se branche dans le monde, pourquoi et à quel prix. Un bon exemple de cette approche figure dans l'article de Sarah Fila-Bakabadio qui s'intéresse à la manière dont les Afro-Américains s'approprient la musique africaine pour les rites néo-traditionnels du Kwanzaa. Ensemble comme séparément, les contributions à ce numéro évoquent une observation importante concernant la musique populaire africaine : il s'agit toujours et déjà de world music, en premier lieu à cause de la manière dont elle s'est trouvée impliquée dans des réseaux de signification qui semblent toujours se trouver à peine hors de ses frontières.

Comment les auteurs sont-ils parvenus à cet objectif ? D'abord, ils insistent sur l'importance des publics. Non pas des publics de masse au sens d'Adorno, mais des publics délicats et souvent critiques qui, de manière quelque peu surprenante, participent à la production du sens musical et pour qui la musique peut être autant une source de tension sociale que la marque d'une identité de groupe (Fila-Bakabadio ce numéro). Dorothea Schulz nous offre une réflexion extrêmement riche sur la manière dont les différents types de publics se branchent sur différents types de médias. Nous voyons 
en particulier comment les jeunes femmes du milieu urbain au Mali expriment une préférence pour les «stars » locales - ce qui n'est généralement pas le cas pour les hommes - et comment les notions de beauté et d'honneur sont liées au cosmopolitisme des emblèmes de consommation. Mais son article évoque également comment la musique populaire donne à ces jeunes femmes « un accès imaginaire au monde de la réussite et de la fierté féminine dont elles se sentent exclues ». Dans son article sur la musique populaire et la culture urbaine à Abidjan, Yacouba Konate nous donne une occasion bien rare d'apercevoir ce qui différencie les différents publics de jeunes ( « ... La conjonction entre l'incertitude des futures élites et la désillusion des exclus du système...»), mais il suggère également le potentiel qu'ont les publics de constituer un champ de signification sociale et politique. Bob White se penche sur la manière dont les préférences des publics urbains au Congo créent des espaces pour des cosmopolitismes qui, pour diverses raisons, sont plus attirants que ceux façonnés par le stoïcisme du régime colonial. La contribution de Brian Larkin sur les films indiens et la musique bandiri à Hausaland montre les réactions des publics non pas comme des moments de résistance mais comme des instants d'aspiration cosmopolite. Ce qui paraît le plus central dans son analyse, c'est la manière dont cette forme musicale mimétique (dans laquelle des chansons de films importés sont adaptées pour être utilisées dans la musique dévotionnelle locale) creuse un fossé entre des visions concurrentes de la piété islamique.

Les articles de ce numéro attachent beaucoup d'importance au concept d'intermédiaire culturel (cultural brokerage), mais ce n'est que récemment que les anthropologues ont pu surmonter le malaise profond qui accompagne l'étude de ceux qui cherchent à se situer stratégiquement entre les savoirs partiels des autres. Les intermédiaires culturels peuvent être des empresarios dans le cas des marchands grecs qui ont joué un rôle déterminant dans l'émergence de la musique congolaise (White ce numéro), ou simplement des experts en musique qui sont engagés par divers commanditaires officiels et institutionnels (comme dans les cas présentés par Francis Falceto et Denis-Constant Martin, dans ce numéro). Et, très souvent, les musiciens africains sont des intermédiaires culturels à part entière (Falceto 2001; Hayward 1998 ; Konate 1987 ; Veal 2000). Youssou N'dour est connu comme étant «l'enfant prodige » de Peter Gabriel, mais il est aussi au centre d'une scène musicale locale à Dakar qui comprend des studios d'enregistrement, des sociétés de production et des boîtes de nuit. Oumou Sangare fait la promotion de la musique populaire de saveur traditionnelle, mais elle porte aussi le flambeau des droits de la femme dans son pays natif, le Mali (Eyre 2000). Thomas Mapfumo, connu surtout pour avoir rendu populaire une forme modernisée de musique « traditionnelle » zimbabwéenne (chimurenga), a aussi ouvert la route à une nouvelle génération de célébrités rebelles (Turino 2000). Et la liste continue: Salif Keita, Geoffrey Oryema, Lokua Kanza, Papa Wemba, Cesaria Evora, Cheb Mami, Khaled..., d'artistes qui sont des stars à l'étranger, et dont les efforts pour s'inscrire dans le 
cosmopolitisme font d'eux des intermédiaires — voire des esclaves — de leurs identités régionales ou nationales.

Ce qui est potentiellement le plus remarquable dans la musique populaire africaine de la fin du $\mathrm{XX}^{\mathrm{e}}$ siècle, c'est la manière dont elle nous ramène à la question de l'identité nationale. Ce retour a jusqu'à récemment été hésitant, soit parce que l'idée d'étudier les cultures nationales semblait insurmontable, soit parce qu'on a trop souvent vu ressurgir le mal au nom du nationalisme. Il reste qu'un certain nombre d'études récentes ont commencé à éclaircir les liens qui existent entre la musique populaire et le nationalisme en Afrique et aux Caraibes (Askew 2002; Averill 1997; Moore 1997 ; Wade 2000, White à paraître). Dans cette même optique, Thomas Turino (2000) nous a démontré que le nationalisme africain tend à aller de pair avec le cosmopolitisme africain plutôt que de s'opposer à lui. Pour certains en Afrique, il est urgent de promouvoir une identité musicale nationale, non seulement pour revendiquer un espace politique neutre, loin des menaces $\mathrm{du}$ tribalisme et des divisions ethniques («...Comment peut-on chasser de l'étranger le jour et consommer de l'étrangère la nuit ? ", Konate ce numéro), mais aussi pour revendiquer un espace dans l'arène politique internationale qui leur a été interdite ou enlevée. La nouvelle monographie de Kelly Askew donne les outils pour comprendre la relation entre la «performance » et la politique. Le message qui transparaît dans l'introduction de son livre est non seulement que la politique est spectaculaire, mais aussi que la culture est produite par le spectacle de la politique

Le compte rendu de Francis Falceto sur le couvre-feu de 18 ans dans l'Éthiopie de l'après-Derg illustre l'importance de la relation entre musique populaire et mémoire collective. En plus de cet essai, Francis Falceto a également produit une série de onze $\mathrm{CD}$ consacrée au travail du visionnaire de la nouvelle vague musicale éthiopienne Amha Eshèté (Buda Music, Paris). Cette série est devenue une collection de référence dans l'histoire immédiate de l'Éthiopie et se présente sous forme d'incarnation, voire récupération, de la mémoire perdue. La mémoire est également un thème central de la contribution de Denis-Constant Martin qui, d'une manière quelque peu similaire à ce que Yacouba Konate a décrit dans le cas d'Abidjan, est confronté à une scène musicale esthétiquement dynamique et historiquement riche n'ayant pas de son unificateur: « À la différence de Rio de Janeiro, de Port of Spain, ou de la Nouvelle Orléans, le Cap n'a pas produit un genre musical susceptible de symboliser son histoire et ses particularités » (Martin ce numéro). Au contraire, comme le montre Martin, ce que l'on voit émerger est une forme de «créolité » musicale, un genre qui s'adapte plus ou moins maladroitement à l'identité musicale articulée par d'autres genres «nationaux».

Certains lecteurs seront peut-être surpris d'apprendre jusqu'à quel point ces divers genres nationaux en Afrique ont subi l'influence de la musique de ce que Paul Gilroy a baptisé «l'Atlantique noire »: le jazz et le gospel (Martin ce numéro), le rhythm \& blues (Falceto ce numéro) et la rumba 
(White ce numéro), pour ne citer que quelques exemples. L'ouvrage récent de Manthia Diawara (1998) nous livre un compte rendu personnel et fascinant de la manière dont la musique et la culture afro-américaines ont permis aux jeunes Africains de faire l'expérience de ce qu'il appelle le homeboy cosmopolitanism (le cosmopolitisme du quartier). On peut se réjouir que les contributeurs à ce numéro spécial ne se soient pas contentés de simplement observer cet héritage musical, mais qu'ils se soient efforcés, chacun à leur manière, de montrer comment ces influences sont incorporées et refaçonnées pour créer de nouvelles formes d'action politique et de nouveaux paysages sonores. Quel meilleur exemple de cela existe-t-il que le rap, qui est sans doute la plus mondiale des world musics, et l'un des genres populaires les plus dynamiques sur le continent aujourd'hui ? ${ }^{1}$ L'essai de Hadj Miliani sur la musique rap et la culture des jeunes en Algérie tente d'expliquer comment cette forme d'expression mondiale est adaptée à l'usage local et comment, à beaucoup d'égards, elle contribue à redéfinir les notions traditionnelles d'espace public. Si le groupe de rap congolais Bisso na Bisso a été acclamé pour avoir produit l'un des albums les plus vendus en France en 1999 (Elbadawi 1999: 48), ce n'est pas parce qu'il était moins «traditionaliste » que Positive Black Soul (groupe basé à Dakar et considéré pendant des années comme le représentant inégalé du hip-hop africain), mais simplement parce qu'il était plus «world».

Quand les «musiques nationales » tentent d'effectuer le saut potentiellement périlleux dans la «world music », le cadre discursif du genre (et bien souvent l'identité de son créateur) devient inévitablement enfermé dans l'une ou l'autre des deux formes de langage, voire dans les deux. Le premier est un langage de l'universel, platitude qui caractérise toute musique qui entreprend de traverser les frontières. C'est la vision utopique de la communauté qui est le produit d'un effacement naïf : «Nous sommes tous pareils, tralala, la musique est une langue universelle, tralala, quelle que soit la couleur de ta peau, tralala, nous sommes tous pareils, trala lalala... » Dans le contexte de la world music, cette stratégie a fait ses preuves envers ce public le plus souvent progressif et remarquablement homogène qu'est le club des consommateurs de world music (White 2000). Le second langage est celui de l'hybride. Il apparaît généralement quand l'auteur articule une identité à la fois traditionnelle et moderne, le meilleur exemple étant l'identification simultanée de Marie Daulne aux "pygmées d'Afrique centrale » et aux «ethnomusicologues dont les disques m'ont inspirée » (Zap Mama, Adventures in Afropea 1, Luaka Bop 1993). Les notes qui figurent sur la jaquette de cet album expliquent les sources d'inspiration pour chaque chanson, mais cette honnêteté intellectuelle masque un autre ensemble de stratégies : « ... Invoquer l'hybridité comme sa propre position identitaire, donne

1. Pour un excellent aperçu du hip-hop en Afrique sub-saharienne, voir le site www.africanhiphop.com. 
alors le droit de revendiquer l'utilisation de l'intégralité du terrain spatiotemporel de cette identité comme une palette artistique » (Feld 1996: 2).

Il s'avère que cette tension entre unité et différence est précisément le langage utilisé par les musiciens africains qui entreprennent de représenter l'Afrique, ou une partie de l'Afrique, par la chanson. Un inventaire rapide de la world music africaine produite au cours des dix dernières années fait apparaître un nombre surprenant de chansons consacrées à l' «Afrique » ou contenant le mot «Afrique» dans le titre ou dans les paroles. En effet, la référence à l'Afrique est devenue une norme dans une grande partie du répertoire populaire africain, qu'il appartienne ou non à la world music. Les chansons sur l'Afrique mêlent généralement les langages national et colonial, suggérant ainsi la pluralité des publics concernés par ce message, et un nombre important de chansons ne font qu'énumérer les noms de villes africaines ou de pays africains (Africa by Night de Bisso na Bisso en est un bon exemple récent). Il est tentant de dire que cette tendance marque l'existence d'une certaine re-territorialisation, mais, à y regarder de plus près, nous constatons que cette énumération s'insère dans des discours d'unité quelque peu liée au passé héroïque de la lutte panafricaniste...

« Change your thinking, walk together, keep on working, Cheikh Ante Diop, Kwame Nkrumah, Stephen Biko, all you people, Africa...Africa unite» (Youssou N'dour, New Africa)

... et qui semble rêver à un passé-futur composé qui ne serait plus la victime des frontières territoriales que l'on pense être à l'origine de tant de tragédies humaines :

«The Senegal that I love so...Weren't you once free my Senegal ? Long ago, long ago, long ago...why oh why... ? » (Wasis Diop, African Dream, No Sant, Mercury Records, 1996).

Dans la jaquette de son récent album Baloba!, le groupe Africando (Sénégal) rend explicite le lien entre l'esclavage, la mémoire et la notion transcendante d'une «Grande Afrique » transatlantique :

«En ce $150^{\mathrm{e}}$ anniversaire de l'abolition de l'esclavage, l'auteur rappelle à la communauté afro-carabéenne son devoir de mémoire, par le biais de son art, il relate les origines de la musique afro-cubaine. Souvenez-vous, frères, que cette musique "trait d'union" entre nos peuples, a pour source La Grande AFRIQUE ! Ce fruit, mélange de rythmes Ghanéen, Congolais, des langues Yoruba et Espagnoles, émane de l'histoire d'esclavage. Histoire des Noirs de la côte d'Afrique qui on transité du Dahomey (Bénin) au Sénégal ; par la plage de Djêbadji ville de Ouidah à l'île de Gorée, vers les Antilles, les Caraïbes, Cuba jusqu'en Amérique ».

(Résumé de la chanson Huenouhwo Stern's 1998).

Malheureusement, pour Africando, ce type de rappel historique se vend mal. Comme 1'a montré Denis-Constant Martin (1996), la world music est 
un rêve, «mais ce rêve est aussi un marché ». C'est pourquoi le véritable hymne du continent est probablement plus proche d'une version banalisée de l'Afrique où les gens mangent à leur faim, travaillent dur et ont la danse « dans le sang ».

« Ça fait rêver, ça fait vibrer, ça fait danser, oh Africa...

C'est sa façon, bosser beaucoup, manger beaucoup, oh Africa...

C'est sa fort (sic), manger beaucoup, danser beaucoup, oh Africa...

Il y a tekere, il y a dolé, il y a foutou, il y a foufou, il y a noko, oh quel bon manger... Il y a dédé, il y a yassa, il y a mafé, efanko, il y a coco, oh quel bon manger... » (Salif Keita, Africa, Mango Records, 1995).

La plus grande partie des écrits consacrés à la musique africaine ne fait que reproduire le type de clichés journalistiques qui ne nous disent rien de substantiel sur la culture ou sur la politique africaine: «En Afrique, la musique est partout »... «En Afrique, la musique accompagne tous les moments importants de la vie et de la mort »... «En Afrique, la musique est une forme de résistance »... «En Afrique, la musique est une sorte d'échappatoire », etc. Dans la reprise de ses travaux sur la world music, Denis-Constant Martin (2002) ramène le lecteur à une question essentielle : la world music doit-elle être lue comme une forme d'engagement ou comme une forme de dénégation? À mes yeux, il s'agit là d'une question urgente et brûlante, particulièrement en ce qui concerne l'Afrique qui, plus que tout autre continent, a souffert des conséquences de l'appropriation, et pas uniquement dans le domaine de la musique. Ces quelques observations préliminaires présentées ici suggèrent différents chemins entre la production culturelle et la politique de la culture en Afrique contemporaine. Les articles éminemment riches regroupés ici dans ce numéro spécial nous montrent non seulement comment l'histoire se répète, mais aussi de quoi l'histoire — et le monde — sont faits.

Université de Montréal.

\section{BIBLIOGRAPHIE}

AMSElle, J.-L.

2001 Branchements. Anthropologie de l'universalité des cultures, Paris, Flammarion.

Appadurai, A. (ed.)

2000 Globalization, Durham, N.C. Duke University Press. 
AsKew, K. M.

2002 Performing the Nation: Swahili Music and Cultural Politics in Tanzania, Chicago, The University of Chicago Press.

Attali, J.

1977 Bruits. Essai sur l'économie politique de la musique, Paris, PUF.

AVERILl, G.

1997 A Day for the Hunter, A Day for the Prey: Popular Music and Power in Haiti, Chicago, The University of Chicago Press.

Born, G. \& Hesmondhalgh, D.

2000 Western Music and Its Others : Difference, Representation and Appropriation in Music, Berkeley, University of California Press.

ChARry, E. S.

2000 Mande Music: Traditional and Modern Music of the Maninka and Mandinka of Western Africa, Chicago, University of Chicago Press.

DiAWARA, M.

1998 In Search of Africa, Cambridge, MA, Harvard University Press.

Elbadawi, S.

1999 «L'aller-retour de Bisso na Bisso », Africultures, 21 : 45-48.

ERLMANN, V.

1993 «The Politics and Aesthetics of Transnational Musics», The World of Music, 35 (2) : 3-15.

1996 « The Aesthetics of the Global Imagination : Reflections on World Music in the 1990s », Public Culture, 8 : 467-487.

1999 Music, Modernity and the Global Imagination, New York, Oxford University Press.

EYRE, B.

2000 In Griot Time : an American Guitarist in Mali, Philadelphia, Temple University Press.

FALCETO, F.

2001 Abyssinie Swing : a Pictorial History of Modern Ethiopian Music, Addis Ababa, Shama Books.

FELD, S.

1996 «Pygmy POP : a Genealogy of Schizophonic Mimesis », Yearbook for Traditional Music, 28 : 1-35.

2000 «A Sweet Lullaby for World Music», in A. ApPADuraI (ed.), Globalization, Durham, N.C., Duke University Press : 189-216.

Guilbault, J.

1993a « On Redefining the "Local" Through World Music », The World of Music, 35 (2) : 33-47. 
1993b Zouk: World Music in the West Indies, Chicago, University of Chicago Press.

1996 Beyond the «World Music » Label : An Ethnography of Transnational Musical Practices. Disponible sur l'internet : http://www2.rz.hu-berlin.de/fpm/ texte/guilbau.htm.

НАмм, С.

1995 Putting Popular Music in Its Place, New York, Cambridge University Press.

Hardt, M. \& Negri, A.

2000 Empire, Cambridge, MA, Harvard University Press.

HAYWARD, P.

1998 Music at the Borders : Not Drowning, Waving and their Engagement with Papua New Guinean Culture (1986-1996), London, John Libbey.

HeRnandez-Reguant, A.

2000 Marketing Cuban Music and the Buena Vista Social Club, Présentation donnée au congrès IASPM et SEM, Toronto, Canada.

KeightLy, K.

1998 Reading Capitol/EMI : Musical Tourism and Industrial Globalization in the Record Industry, 1954-1963, Présentation donnée au congrès IASPM-USA, UCLA, 16 octobre : 1-11.

Konate, Y.

1987 Alpha Blondy: Reggae et société en Afrique Noire, Paris, Karthala.

MANUEL, P.

1993 Cassette Culture: Popular Music and Technology in North India, Chicago, The University of Chicago Press.

Martin, D.-C.

1996 «Qui a peur des grandes méchantes musiques du monde», Cahiers de Musiques traditionnelles, 9: 3-20.

2002 «Les "musiques du monde": imaginaires contradictoires de la globalisation », in D.-C. MARTIN (ed.), Sur la piste des OPNI (Objets politiques non identifiés), Paris, Karthala.

MEINTJES, L.

1990 «Paul Simon's Graceland, South Africa, and the Mediation of Musical Meaning », Ethnomusicology, Winter issue : 37-73.

Moore, R.

1997 Nationalizing Blackness : Afrocubanismo and Artistic Revolution in Havana, 1920-1940, Pittsburgh, University of Pittsburgh Press.

PACINI-HeRnANDEZ, D.

1993 «A View from the South : Spanish Caribbean Perspectives on World Beat», The World of Music, 35: 48-69. 
PekKa, G. \& SAunio, I.

1998 An International History of the Recording Industry, New York, Cassel.

TAYLOR, T. D.

1997 Global Pop : World Music, World Market, New York, Routledge.

TURINO, T.

2000 Nationalists, Cosmopolitans, and Popular Music in Zimbabwe, Chicago, University of Chicago Press.

VEAL, M. E.

2000 Fela: The Life and Times of an African Musical Icon, Philadelphia, PA, Temple University Press.

WADE, P.

2000 Music, Race and Nation : Musica Tropical in Colombia, Chicago, University of Chicago Press.

White, B. W.

2000 «Soukouss or Sell-Out? Congolese Popular Dance Music on the World Market », in A. Haugerud, M. P. Stone \& P. D. Little (eds), Commodities and Globalization: Anthropological Perspectives, New York, Rowman \& Littlefield : 33-58.

À paraître Ndule Popular Culture and the Politics of Dance Music in Mobutu's Zaire, Durham, N.C., Duke University Press.

ZEMP, H.

1996 «The/An Ethnomusicologist and the Record Business », Yearbook for Traditional Music, 28 : 36-56. 